\title{
Satiation effects in the perception of single letters'
}

\author{
JAMES R. POMERANTZ, STEPHEN KAPLAN ${ }^{2}$ AND RACHEL KAPLAN
}

UNIVERSITY OF MICHIGAN

\begin{abstract}
When single letters were presented for five flashes, with $S$ making a clarity judgment after each exposure, initial potentiation and subsequent satiation effects were found. $A$ processing task and complete prior knowledge led to an increased probability of a "clear" response on the first exposure and to an earlier onset of the satiation effect. The results are discussed in terms of the neural network model underlying this research.
\end{abstract}

Many tachistoscopic experiments have been concerned with the facilitating role of such variables as exposure duration, frequency, illumination, contrast, and meaning on stimulus threshold. Haber and Hershenson (1965), for example, showed that repeated exposures of constant duration can also have a powerful effect on threshold and stimulus clarity. Other recent studies, on the other hand, have demonstrated the decline of percept clarity with repeated or prolonged exposures. McKinney (1966) has shown the "fragmentation" of simple figures with long unstopped exposures. In the auditory modality Warren and Gregory (1958) have reported the distortion of words with repeated oral presentations. Haber and Hillman (1966), in another tachistoscopic experiment designed to reveal the effects of repeated exposures on the growth of single-letter percepts, indicated that similar fragmentation effects may appear with as few as five exposures.

These studies suggest that the effect of such parameters as stimulus duration and repetition may not be unidirectional and may in fact serve to elicit two opposing functions. The first of these, responsible for percept growth, is labeled potentiation; the second function, associated with decline in the strength of clarity of the percept, is called satiation.

Haber's discussion of his own studies has concentrated almost exclusively on the potentiation effect of repeated exposure, i.e., the growth of percepts with repetition (Haber, 1965; Haber \& Hillman, 1966). He has tended to describe his results in terms of monotonically increasing functions and to discount the satiation effects cited above as artifactual. In reviewing Haber's experiments, Neisser (1967) has claimed that the observed potentiation is attributable to the perceiver's growing set of expectancies with repeated exposures. Having processed a portion of the information present in the stimulus on one exposure, the $S$ expects that same information to be present in the next exposure; therefore, Neisser argues, processing time required for that information on the second exposure is reduced, and additional information can be assimilated. This account, like Haber's, is unable to accommodate the phenomenon of satiation, unless expectancy is considered to have an inhibitory as well as facilitatory role.

Nevertheless, there is reason to suspect that the satiation effects observed in the Haber and Hillman study are real though of small stature. If the same perceptual mechanisms operating in McKinney's fragmentation study are involved in processing brief, repeated exposures, then perhaps fragmentation or satiation effects are also to be expected. A recently developed neural circuit model (Kaplan, 1968) contains in its account of the phenomenon of perceptual growth with repeated brief exposures a prediction of a satiation effect of this kind. This model is basically Hebbian in nature, incorporating also Good's (1965) concept of subassemblies-small tightly connected groups of neurons. The model is built on four basic constructs: reverberation, short-term memory, fatigue, and long-term memory. With these it attempts to account for a wide range of data including the reminiscence and inaccessibility findings by Kleinsmith and Kaplan (1963, 1964).

The long-term memory construct plays no central role in the present context. Of the others, reverberation refers to neural activity within a closed loop or lattice of interconnected neurons and does not itself serve as short-term memory in this model. Although opposite in effect, short-term memory and fatigue follow similar rules. They both increase as a function of reverberation and they both decay during a period of rest. Fatigue, however, shows a considerably more peaked distribution over time; it takes longer to accrue and is faster to decay. These assumptions yield a model of reverberation in which an initial positive feedback phase in time gives way to a negative feedback phase, effectively terminating circuit activity. Reverberation increases short-term memory, which, in turn, facilitates reverberation. In the long run, however, reverberation, since it is based on repeated firing, involves the expenditure of resources that leads inevitably to fatigue.

In terms of this model the effect of repetition on the clarity of a percept can be conceptualized as follows: Each brief exposure excites a small number of subassemblies. A residue of short-term memory remains after each exposure. Repeated presentations result in a wide sampling of relevant subassemblies being stimulated, with some being excited on more than one occasion. With repetitions, this may lead to sufficient summation to generate activity in a larger circuit. At the same time, as the reverberation progresses, fatigue effects will also be expressed leading to a decline in percept clarity. (Though the fatigue will dissipate with time, this aspect is not a major concern in the present study.)

The present experiments were undertaken to test the hypothesis that the development of percept clarity with repeated exposures will take the form of an inverted $U$ rather than a simple monotonically increasing relationship. A task involving prior processing of the critical stimuli would be expected to have an effect similar to repeated presentation but perhaps of greater mangitude. It was thus predicted that a processing task prior to the repeated exposures would lead to stronger satiation effects for the "preprocessed" items.

\section{Method}

\section{EXPERIMENT 1}

Subjects. The Ss were 12 female University of Michigan undergraduates drawn from the unpaid introductory psychology subject pool. Each was tested individually in two $1.5 \mathrm{~h}$ sessions.

Stimuli. The stimuli were 18 letters from the English alphabet. Because of width consideration or possible ambiguity, I, L, M, O, $Q, W, Y$, and $Z$ were not used. The black stimuli, drawn from Letraset Sheet 192, were affixed to plain white bristol board sheets.

The stimuli were presented in one channel of a two-channel mirror tachistoscope. The second channel, containing a penciled rectangle $1 \times 3 / 4$ in. for a fixation boundary, served as background and was lighted at all times when the stimulus channel was unlighted. The visual angle of the stimuli was $17 \mathrm{~min}$. Reflectance 
measures at the eyepiece was $.5 \mathrm{ft}-\mathrm{L}$ for the background and stimulus channels.

Each letter was presented either two, four, or five times and then was changed to another letter. The interexposure interval for a given letter was approximately $1 \mathrm{sec}$ while the interval between letters, i.e., after the last exposure in a series for a particular letter, was approximately $4 \mathrm{sec}$.

The duration of each exposure was kept constant within each session and was determined for each $S$ at the beginning of the session. Ascending and descending method of limits was used to obtain that exposure time at which $S$ had a .5 probability of correctly identifying an unknown stimulus on one exposure.

Processing task. The S was told two target letters and immediately began a 1-min search through sheets containing random stimuli, circling either target whenever she came across it.

Stimulus orders. Twelve lists were used with half the Ss receiving six of these and the other half the other six. Each list contained each of the 18 letters once. It was constituted as follows: For each set of three consecutive letters, one was presented for two exposures, one for four, and one for five; two were the target stimuli from the immediately preceding processing task and one was not. Each list was balanced with respect to number of exposures and the position of the target letters from the processing task. Following each set of three tachistoscopically presented letters the processing task was repeated with two new target letters.

Procedure. The $\mathrm{S}$ was instructed to make two responses following each exposure in the tachistoscope. She was to identify the stimulus and to judge the clarity of her percept of the stimulus using the three categories, "nothing" if no part of the stimulus was detected, "vague" if part but not all of the stimulus was perceived, and "clear" if the entire stimulus was perceived.

Each session consisted of three lists with a 10-min break between lists. Each processing task period was followed by three different letters, the two target letters and one different letter. One of the letters was presented for two exposures, one for four, and one for five. Approximately $4 \mathrm{sec}$ after the last exposure of the third letter, two new target letters were announced for the next processing task period.

Scoring. The analyses are based on the trials involving five exposures since the predictions are based on the effect of repeated exposures of the same stimulus. (Using three different sequence lengths prevented $S$ from knowing how many times in succession a particular letter would be presented.) For each of the five expcsures of letters from the processing task and of letters not involved in that manipulation the instances of "clear" and "correct" judgments were computed. There were 24 instances of "preprocessed letters" and 12 instances of "nonprocessed letters" for each $\mathrm{S}$.

\section{Results}

The results are presented in the two lower functions in Fig. 1. The three significant features to be noted are the gradual increase in clarity with repeated exposures $(F=6.75 ; \mathrm{df}=4,44 ; \mathrm{p}<.001)$, the decrease between the fourth and fifth exposures with the "preprocessed letters" ( $\mathrm{t}=2.15 ; \mathrm{df}=11 ; \mathrm{p}<.05)$, and the consistent superiority of the "preprocessed letters" $(F=20.11$; $\mathrm{df}=1,11 ; \mathrm{p}<.001)$.

The first of these is consistent with previous studies already cited and does not warrant special consideration here. The second, however, is more relevant to the present purposes. The decline in "clear" judgments between the fourth and fifth exposures is suggestive of the predicted inverted-U relationship. It should be noted that this decline was found only for the "preprocessed letters."

The third feature, the discrepancy in "clear" judgments

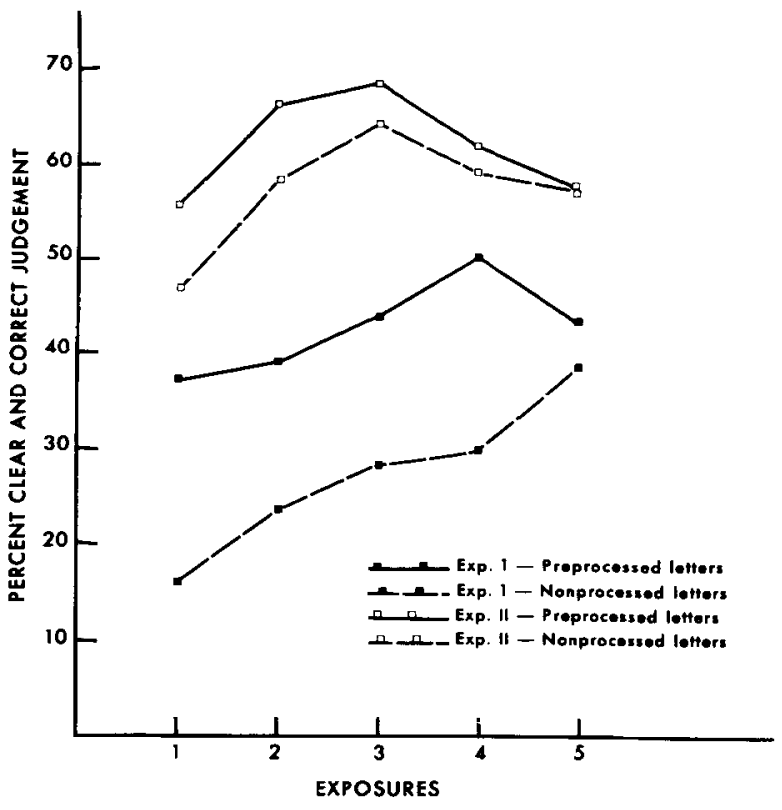

Fig. 1. Probability of "clear" judgment as a function of number of exposures for "processed" and "nonprocessed" letters.

between the "preprocessed" and "nonprocessed" letters is subject to several interpretations. One of these points to a possible methodological flaw, namely, that one difference between the two functions involves the issue of prior knowledge of the stimulus. The comments offered by several Ss testify to the fact that they quickly became aware of the procedure and realized that the preprocessed letters would be two of the three letters following the processing task. Thus the resulting function may have reflected both the partial prior knowledge as well as the processing task. Haber (1965) has shown that prior knowledge of the stimulus to be presented has a potentiating effect on percept clarity. A second experiment was undertaken to determine the role of prior knowledge in this phenomenon.

\section{Method}

\section{EXPERIMENT 2}

Subjects. Ten female University of Michigan undergraduates from the unpaid subject pool served as Ss. None of these had participated in Experiment 1. Each was tested individually in two 1.5-h sessions.

Stimuli and stimulus orders. The same stimuli and lists were used as in Experiment 1.

Processing task and procedure. The processing task again involved two target letters, but $S$ was instructed to repeat these in alternate fashion for $1 \mathrm{~min}$. It was emphasized that steadiness rather than rate in the oral repetition was important.

Immediately prior to the first exposure of each letter, E informed $S$ what the letter would be. Since $S$ thus had prior knowledge of each letter, her response involved only the clarity judgments. In all other respects Experiment 2 followed the same procedure as Experiment 1.

Scoring. Again the trials involving five exposures of the same letter form the basis for the analyses. Since the letters were not identified by $S$, the computations are based only on the probability of a "clear" judgment for each exposure for the "processed letters" and the "nonprocessed letters."

\section{Results}

The top two functions in Fig. 1 represent the results for 
Experiment 2. The effect of number of exposures is again significant $(F=4.01 ; \mathrm{df}=4,36 ; \mathrm{p}<.01)$. The difference between the two functions is not significant $(F=4.69 ; \mathrm{df}=1,9 ; \mathrm{p}<.10)$ and the two functions were therefore combined in further analyses.

The shape of the combined function is as predicted. The "clear" judgments at the third exposure are significantly more probable than at either the first or fifth exposure $(t=7.3$ and 4.2 , $\mathrm{df}=40 ; \mathrm{p}<.05)$, and the two endpoints do not differ significantly from each other. A trend analysis reveals that the function is significantly quadratic $(\mathrm{F}=12.80 ; \mathrm{df}=1,36$ $p<.001)$ and the quadratic component accounts for $80 \%$ of the variance (Winer, 1962).

\section{DISCUSSION}

The main effect of Experiment 2 appears to support the prediction of an inverted-U relationship between number of exposures and the clarity of the percept. Before accepting this prediction as supported, however, the alternative explanations Haber and Hillman offered for the downturn on their Trial 5 should be examined in the context of the present study.

The drop observed in the Haber and Hillman study only appeared on the fifth (and last) trial, and primarily in the first of their two studies. As the authors indicate, Ss often show an improvement in their last trial in a series, apparently because they know it is their last chance to make a report for that item. This effect, of course, is the opposite of the one they observed. An indication in our data that some effect specific to the last trial is not at issue, is the decline in the fourth as well as the fifth exposure in Experiment 2.

Haber and Hilman suggest that an imbalance in their design may be responsible for the fifth-trial decline; the five-exposure trials in their first experiment appeared to have a disproportionate representation of more difficult (i.e., low frequency) letters. This artifact, however, can only arise when different items are used to obtain the effects of varying numbers of presentations. Our Fig. 1 is based on the same items for all points within a given function. In other words, it represents the per cent clear judgments for the first exposure of all five-exposure series, for the second exposure of the same series, and so on. In such a repeated measure or "item as its own control" design, the confounding of item difficulty and number of exposures cannot occur.

It should perhaps be noted in passing that a traditional endeffect, an improvement in the last trial performance, was found in the case of the "nonprocessed letters" in Experiment 1. These items are in certain respects most comparable to the Haber and Hillman trial since neither prior knowledge nor the processing task was used. A major difference, however, lies in the fact that our function is based on 12 triais in two sessions $(N=12)$, while the Haber and Hillman study is based on approximately 16 trials for each of at least six sessions $(\mathrm{N}=6)$ using highly practiced Ss. If the Haber and Hillman data were analyzed by sessions, it is possible that a stronger endeffect would be found for the early sessions.

A striking difference between our two experiments is in the perceived initial clarity of the material. In terms of the model, both the prior knowledge and the processing task tend to produce prolonged activity in the neural circuitry corresponding to the relevant stimuli, resulting in priming or potentiation. In the visual search task, activity must be maintained in the neural circuits processing the target letters in order for a match to be made when the target is encountered. With the oral repetition procedure (Experiment 2), activity in the neural circuits representing the target letters must be maintained for oral response generation and, further, is sustained by the auditory feedback the $S$ receives from his own responses. The potentiating effects of prior knowledge obtain in a similar fashion. The magnitude of neural circuit activity prior to an exposure depends in part on the extent to which the $S$ expects a given stimulus to be exposed. On this point, the neural net model is in full agreement with Neisser; the difference, however, lies in the near omnipotent role Neisser assigns to expectancy and in the failure of his hypothesis to account for satiation.

If indeed the initial level of the function reflects the degree of prior activity in the corresponding neural trace, one might expect some relationship between the initial level and the onset of satiation. Examination of Fig. 1 indicates that this is the case. The number of exposures required for satiation effects to appear is related to the probability of a "clear" response on the first exposure: the "nonprocessed" curve of Experiment 1 has an initial probability of approximately .17 and shows no satiation effects; the "preprocessed" curve of Experiment 1 has an initial probability of .39 and shows satiation effects after the fourth exposure; the initial probabilities for the two functions of Experiment 2 are .47 and .56 and the satiation effects are evident after the third exposure.

It thus appears that prior information or experience will speed the onset of satiation proportional to its initial potentiation effect (in this case the increase in initial clarity). This observation can be seen as a special case of a more general hypothesis: The relationship between experience and availability will approximate an inverted $U$.

The neural net model that gives rise to this hypothesis also points to two important limitations as to the conditions under which effects of this kind are likely to be observed. First, the degree to which the stimulus is impoverished is critical. Impoverishing manipulations, like the very brief durations used in our studies, result in a highly nonredundant stimulus. In theoretical terms, the stimulus is represented by a relatively small number of subassemblies. While the rapid fatigue (and recovery) of a few subassemblies would not be noticed out of the large population of subassemblies representing a redundant stimulus, this loss could be substantial for a minimally represented stimulus. The second limitation concerns the time between stimuli. The inverted $U$ is the outcome of the growth of short-term memory followed by the growth of fatigue (which has a subtractive effect). Both short-term memory and fatigue dissipate with time, with fatigue showing the more rapid decay function. The spacing of trials, then, is an important variable; the longer the interval between presentations, the more likely the fatigue will have dissipated, leaving no evidence in the form of performance decrement. The model thus points to the stimulus duration, the number of trials, the interstimulus interval, and amount of immediately prior experience with the stimulus as critical variables in obtaining this effect.

\section{REFERENCES}

GOOD, I. J. Speculations concerning the first ultraintelligent machine. Advance in Computers, 1965, 6, 31-88.

HABER, R. N. The effect of prior knowledge of the stimulus on word recognition processes. Journal of Experimental Psychology, 1965, 69, 282-286.

HABER, R. N., \& HERSHENSON, M. Effects of repeated brief exposures on the growth of a percept. Journal of Experimental Psychology, 1965, $69,40-46$.

HABER, R. N., \& HILLMAN, E. R. Changes in single letter clarity with repetition. Perception \& Psy chophysics, 1966, 1, 347-350

KAPLAN, S. The formation of a percept: An application of a neural net model. Unpublished manuscript, University of Michigan, 1968.

KLEINSMITH, L. J., \& KAPLAN, S. Paired associate learning as a function of arousal and interpolated interval. Journal of Experimental Psychology, 1963, 65, 190-193.

KLEINSMITH, L. J,, \& KAPLAN, S. The interaction of arousal and recall interval in nonsense syllable paired associate learning. Journal of Experimental Psychology, 1964, 67, 124-126. 
McKINNEY, J. P. Verbal meaning and perceptual stability. Canadian Journal of Psy chology, 1966, 20, 237-242.

NEISSER, U. Cognitive psychology. New York: Appleton-Century-Crofts, 1967.

WARRFN, R. M., \& GREGORY, R. L. An auditory analogue of the visual reversible figure. American Journal of Psychology, 1958, 71, 612-613.

WINER, B. J. Statistical principles in experimental design. New York: McGraw-Hill, 1962.

\section{NOTES}

1. This study is based on an Honors Thesis by the first author. The research was supported, in part, by Research Grant MH-11599 from the National Institute of Mental Health, United States Public Health Service.

2. Address: Department of Psychology, The University of Michigan, Ann Arbor, Michigan 48104.

(Accepted for publication February 19, 1969.) 